ISSN 1112-9867

http://www.jfas.info

\title{
ORGANIC FERTILIZATION OF OLIVE TREE BASED ON SOIL ANALYSIS AND FOLIAR DIAGNOSIS: A CASE STUDY OF AN OLIVE ORCHARD IN THE NORTHWEST OF TUNISIA
}

\author{
K. Ibrahimi ${ }^{1, *}$ and F. Gaddas ${ }^{2}$
}

${ }^{1}$ Institut Supérieur Agronomique de Chott-Mariem. BP 47, 4042 Chott-Mariem, Sousse, Tunisie

${ }^{2}$ Institut National Agronomique de Tunisie. 43 avenue Charles Nicole 1082, Tunis Mahrajène, Tunisie

Received: 23 March 2015 / Accepted: 21 July 2015 / Published online: 1 September 2015

\begin{abstract}
The present study aims at developing an organic fertilization program of an olive orchard located in the North-west of Tunisia. Soil analyses showed an acceptable level of $\mathrm{P}_{2} \mathrm{O}_{5}$ and $\mathrm{K}_{2} \mathrm{O}$ in the soil of the studied farm. Based on leaves and olive fruit analyses, the requirements in $\mathrm{N}, \mathrm{P}$ and $\mathrm{K}$ nutrients of the olive tree for a yield of 20 tons per ha were evaluated at $171 \mathrm{~kg}$ $\mathrm{N} / \mathrm{ha}, 40 \mathrm{~kg} \mathrm{P}_{2} \mathrm{O}_{5} / \mathrm{ha}$ and $170 \mathrm{~kg} \mathrm{~K} \mathrm{~K}_{2} \mathrm{O} / \mathrm{ha}$. As for the required nutrient additions to soil, they were evaluated at $133 \mathrm{~kg} \mathrm{~N} / \mathrm{ha}, 76 \mathrm{~kg} \mathrm{P}_{2} \mathrm{O}_{5} / \mathrm{ha}$ and $264 \mathrm{~kg} \mathrm{~K} \mathrm{~K}_{2} \mathrm{O} / \mathrm{ha}$. In order to satisfy the needs of the olive tree, organic compost was used as soil amendement. The required compost amendments have been evaluated to 4 tons/ha at the age of $3 / 6$ years, to 10 tons/ha at the age of $6 / 10$ years and to 20 tons/ha at an age greater than 10 years.
\end{abstract}

Key words: soil; organic farming; olive tree; foliar diagnosis; fertilization; compost.

Author Correspondence, e-mail: ibrahimi.isacm@gmail.com

doi: http://dx.doi.org/10.4314/jfas.v7i3.2 


\section{INTRODUCTION}

L'agriculture biologique en tant que système de production sain, économique et respectueux de l'environnement a connu dans le monde un progrès considérable durant les dernières années [1]. Ce nouveau concept de production agricole fait actuellement partie intégrante des stratégies développées dans beaucoup de pays où l'opinion publique est devenue de plus en plus consciente de l'importance des produits biologiques [2] [3] [4].

En Tunisie, ce type d'agriculture a touché plusieurs secteurs notamment l'oléiculture qui enregistre ces dernières années une importante évolution. Ainsi, les surfaces cultivées en olivier biologique ont évoluées de 173 ha en 1997 à 13719 ha en 2000 pour atteindre 100500 ha en 2014 [5]. En effet, traditionnellement le secteur oléicole en Tunisie est conduit depuis toujours presque en mode biologique, à part quelques rares administrations d'engrais azotés et quelques traitements fongiques encore plus rares. Il était donc facile de passer au vrai mode biologique.

Une des notions de base de ce nouveau concept de production agricole est la fertilisation biologique visant à maintenir et à développer la fertilité du sol en stimulant ses fractions organique et humique. Cette composante principale de la production biologique reste à nos jours l'une des principales préoccupations des oléiculteurs tunisiens vue l'absence d'études spécifiques en la matière. Ainsi, le présent travail a pour objectif l'élaboration d'un programme de fertilisation biologique raisonnée d'une oliveraie au Nord-Ouest de la Tunisie en se basant sur les analyses du sol et le suivi de l'état nutritionnel de l'olivier par diagnostic foliaire dont plusieurs chercheurs ont montré l'intérêt comme guide de la fertilisation raisonnée de cet arbre [6] [7].

\section{MATERIEL ET METHODES}

\subsection{Zone d'étude}

La zone d'étude est située dans le périmètre public irrigué de Testour relevant du gouvernorat de Beja au Nord-Ouest de la Tunisie (36³1'19.20”N - 9²5'50.00”E) (Fig. 1) Elle appartient à l'étage bioclimatique semi-aride supérieur avec une pluviométrie moyenne annuelle de $375 \mathrm{~mm}$, une température moyenne annuelle de $17^{\circ} \mathrm{C}$ et une évapotranspiration potentielle 
annuelle de $1436 \mathrm{~mm}$. De point de vue géomorphologique, la parcelle d'étude de 5 ha de superficie est située sur un glacis d'accumulation colluvionnaire [8]. La variété d'olive cultivée est la Picholine qui se prête bien aux fortes densités et à une production régulière en Tunisie, aussi bien en conduite irriguée ou en sec [9].

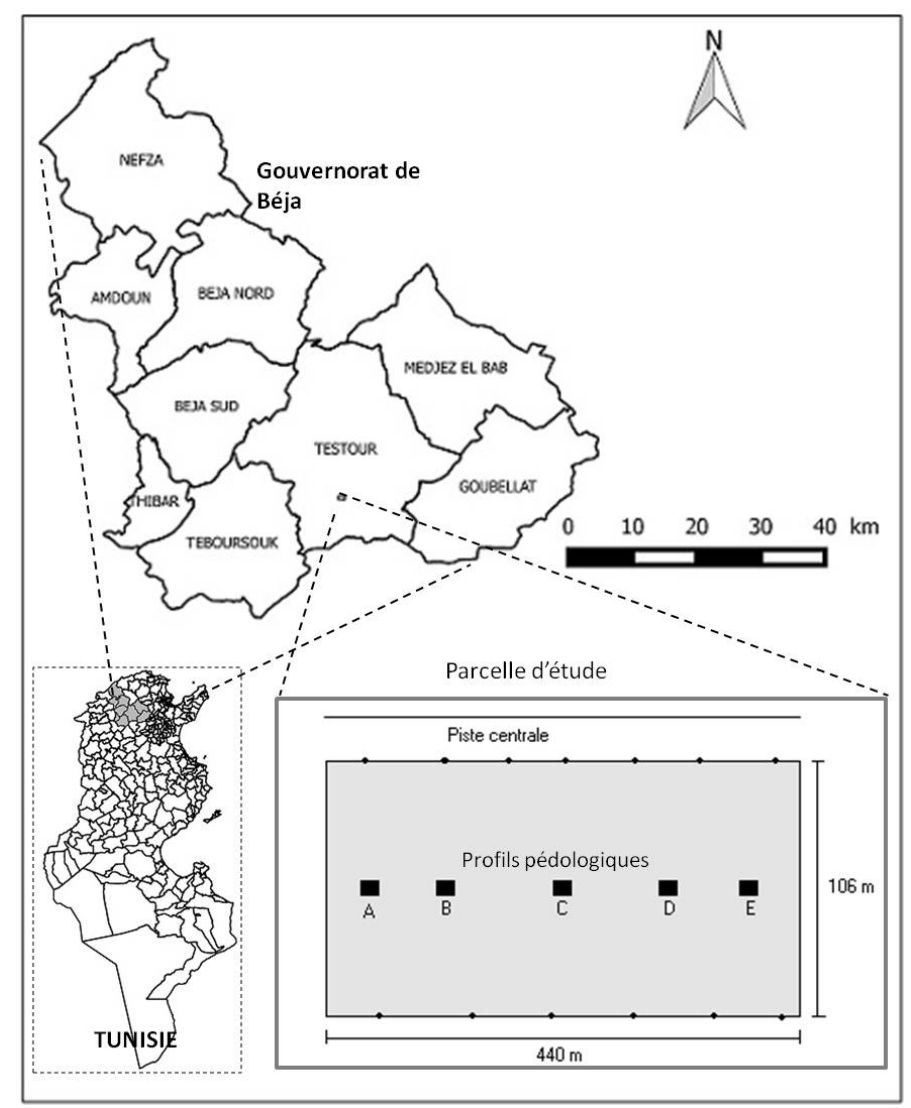

Fig.1. Situation géographique de la zone d'étude.

\subsection{Caractérisation du sol}

Toute la zone d'étude est prospectée, des échantillonnages sont réalisés et cinq profils choisis le long d'un transect amont-aval sont étudiés en détail. Le choix de l'emplacement de ces profils est décidé après une caractérisation préliminaire du sol à la tarière. Des analyses physiques et chimiques ont été réalisées sur les échantillons de sol collectés. L'analyse granulométrique est réalisée par la méthode de sédimentation à la pipette de Robinson [10]. La densité apparente est déterminée par la méthode des cylindres [11]. Les humidités caractéristiques, à savoir la capacité au champ, le point de flétrissement permanent et la 
réserve utile, sont déterminées par la méthode de la chambre à pression [12]. La matière organique (MO) est déterminée par la méthode de Walkley et Black [13]. Le pH est mesuré par un pH-mètre dans une suspension sol/eau. Le calcaire actif est dosé à chaud au $\mathrm{KMnO}_{4}$ [14]. Le phosphore assimilable est déterminé par la méthode d'Olsen et le potassium échangeable à l'aide d'un photomètre à flamme [13].

\subsection{Caractérisation du végétal}

Pour apprécier l'état nutritionnel des oliviers, on a eu recours à la technique du diagnostic foliaire. Ainsi, on a choisi une rangée de 10 oliviers à vigueur moyenne, pour collecter une centaine de feuilles par arbre. On a prélevé les feuilles d'un an situées sur la moitié centrale d'un rameau, tout autour de l'arbre et à hauteur d'homme. L'échantillonnage a été fait à trois reprises durant les mois de février, mars et avril. Les teneurs des feuilles en potassium et en phosphore sont déterminées dans des extraits végétaux respectivement par un photomètre à flamme et par spectrophotométrie. Le dosage de l'azote est réalisé par la méthode Kjeldhal [13]. Un échantillon d'olive a été collecté afin de déterminer sa teneur en N, P et K et avoir ainsi une idée sur l'exportation de ces éléments par $\mathrm{kg}$ d'olives.

\subsection{Caractérisation du compost}

Le compost utilisé comme fertilisant biologique se compose de $50 \%$ de grignon d'olive (débris de coque des noyaux des olives + pulpe d'olive), $30 \%$ de fumier bovin et $20 \%$ de fiente de volaille. Ces proportions sont choisies à la suite de recommandations des services agricoles de la région. La procédure de compostage commence par la mise en couches de différents constituants du compost. Après deux mois, ces couches sont coupées en tranches verticales, mélangées et mises en tas allongés. Ces tas sont entretenus par mélange continu et par un arrosage par semaine pendant quatre mois. Ils sont couverts pour les protéger contre le soleil et les variations de température.

$\mathrm{Au}$ niveau du tas de compost, on a pris dix échantillons sur une profondeur de $50 \mathrm{~cm}$. Par tamisage, on a séparé les deux composantes : débris de coque d'olive et les autres particules. Les analyses ont été effectuées sur un échantillon complet non tamisé et sur les fractions 
tamisées pour déterminer leurs teneurs en N, P et K. Ces analyses ont été faites de la même manière que pour les échantillons du végétal.

\section{RESULTAS ET DISCUSSION}

\subsection{Sol, plante et compost}

En se référant à la classification française [15], le sol de la parcelle d'étude est classé sous la classe des sols calcimagnésiques, sous-classe : carbonatés, groupe: brun calcaire à encroûtement calcaire. Ce sol possède une texture limono-argileuse avec en moyenne $36 \%$ d'argile, $46 \%$ de limon et $18 \%$ de sable. Il présente un taux de calcaire actif très élevé au niveau de l'horizon de surface (28\% en moyenne). Il est aussi caractérisé par un pH alcalin (8,7 en moyenne). De telles conditions peuvent favoriser l'insolubilisation des engrais phosphatés [16], modifier l'activité des micro-organismes du sol [17] et diminuer la stabilité des chélates naturels et artificiels.

Ce sol présente un taux de $\mathrm{K}$ échangeable de 300 ppm donc un niveau de $\mathrm{K}_{2} \mathrm{O}$ de 360 ppm. Selon les normes d'appréciation de la fertilité potassique des sols citées par [18], ce sol présente un taux satisfaisant en $\mathrm{K}_{2} \mathrm{O}$ échangeable. Ce taux de $\mathrm{K}_{2} \mathrm{O}$ échangeable se trouve supérieur aux seuils critiques de cet élément définis par plusieurs auteurs dans les sols à olivier ([19] : 40 à 250 ppm ; [20] : 180 ppm, extraction à l'acétate d'ammonium). Le taux de $\mathrm{P}$ assimilable dans ce sol est de 20,6 ppm donc un niveau de $\mathrm{P}_{2} \mathrm{O}_{5}$ de $47 \mathrm{ppm}$. Ce taux de $\mathrm{P}_{2} \mathrm{O}_{5}$ se trouve proche du seuil de cet élément établi dans les sols à olivier par [21] qui est de l'ordre de 50 ppm (méthode non citée). Il se trouve largement supérieur au seuil proposé par [20] qui est de 18 ppm (méthode Olsen).

Les caractéristiques analytiques des différents profils montrent des différences d'un profil à un autre. En effet, au niveau de l'horizon de surface la teneur en $\mathrm{P}_{2} \mathrm{O}_{5}$ assimilable est de 30,1 ppm dans un profil alors qu'elle est à 9,67 ppm dans un autre. Le taux de matière organique varie de $1,49 \%$ à $1,79 \%$. Une autre différence remarquable d'un profil à un autre est l'épaisseur du sol. Cette profondeur augmente au fur et à mesure qu'on descend la pente. Toutes ces différences montrent qu'un profil moyen (représentant les moyennes des différents critères analysés) cache les faibles teneurs enregistrées dans certaines zones de la parcelle. C'est de ces teneurs faibles qu'il faut tenir compte en vue d'améliorer la fertilité du sol. Pour 
cette raison, on a choisi le profil présentant les teneurs les plus faibles en $\mathrm{P}, \mathrm{K}$ et $\mathrm{MO}$ et on a pris en considération ses teneurs pour l'ensemble de la parcelle (Tableau 1).

Tableau 1. Caractéristiques analytiques du profil de sol considéré comme référence pour

l'ensemble de la parcelle d'étude.

\begin{tabular}{ccccccccccccc}
\hline $\begin{array}{c}\text { Prof. } \\
(\mathrm{cm})\end{array}$ & $\begin{array}{c}\left.\mathbf{d a} / \mathrm{cm}^{3}\right) \\
(\%)\end{array}$ & $\begin{array}{c}\mathbf{L} \\
(\%)\end{array}$ & $\begin{array}{c}\mathbf{S} \\
(\%)\end{array}$ & $\begin{array}{c}\boldsymbol{\Theta}_{\mathrm{cc}} \\
\left(\mathrm{cm}^{3} / \mathrm{cm}^{3}\right)\end{array}$ & $\begin{array}{c}\boldsymbol{\Theta}_{\text {pfp }} \\
\left(\mathrm{cm}^{3} / \mathrm{cm}^{3}\right)\end{array}$ & $\begin{array}{c}\text { RU } \\
(\mathrm{mm})\end{array}$ & $\begin{array}{c}\text { MO } \\
(\%)\end{array}$ & $\mathbf{C a A}$ & $\mathbf{p H}$ & $\mathbf{P}$ & $\mathbf{K}$ \\
\hline $0-30$ & 1,1 & 40 & 39 & 21 & 0,27 & 0,14 & 39 & 1.49 & 26 & 8,65 & 9,67 & 240 \\
\hline
\end{tabular}

Les résultats du diagnostic foliaire effectué dans cette étude sont présentés dans la figure 2. Si l'on compare les teneurs foliaires en $\mathrm{N}$ enregistrées pendant la période de l'échantillonnage aux données de la littérature ([6]), il apparaît que les taux d'azote restent dans les limites normales. De même, si l'on compare les teneurs foliaires en $\mathrm{P}$ aux seuils critiques établis par différents auteurs ([19] : 0,085\% MS ; [6] : 0,05-0,07\% MS ; [20] : 0,07\% MS), il apparaît que tous les échantillons étudiés présentent un taux acceptable de phosphore dans les feuilles. En ce qui concerne le potassium, la comparaison des teneurs foliaires en $\mathrm{K}$ enregistrées pendant cette période aux normes proposées par différents auteurs ([19] : 0,30\% de MS et [20] : 0,5\% de MS) montre qu'aucun des échantillons étudiés n'est carencé en $\mathrm{K}$.

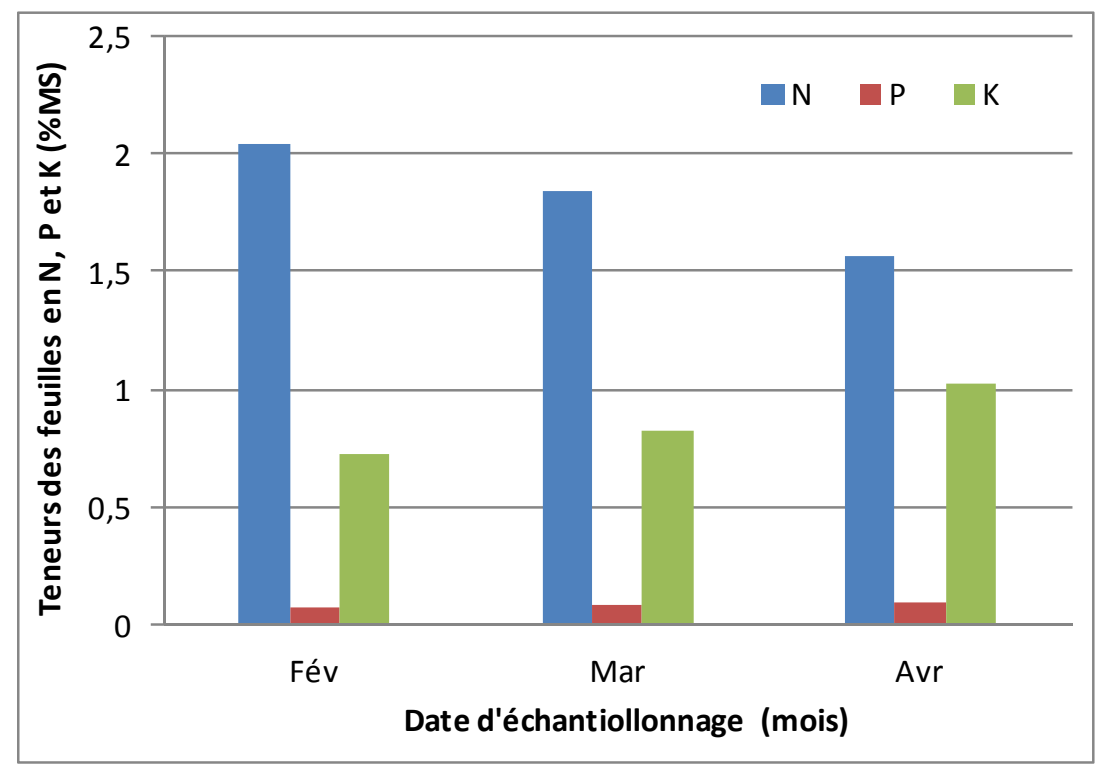

Fig.2. Teneurs des feuilles en $\mathrm{N}$, P et $\mathrm{K}$ (en \% de matière sèche)

Le tableau 2 présente les teneurs en $\%$ de matière sèche et celles par kg d'olive en $\mathrm{N}_{2} \mathrm{P}_{2} \mathrm{O}_{5}$ et 
$\mathrm{K}_{2} \mathrm{O}$ de l'échantillon de fruit d'olivier analysé. En comparant ces résultats aux données de la littérature il apparaît que ces exportations par les fruits de la variété Picholine se rapprochent de ceux enregistrés pour d'autres variétés cultivées dans différentes régions.

Tableau 2. Teneurs en $\mathrm{N}, \mathrm{P}_{2} \mathrm{O}_{5}$ et $\mathrm{K}_{2} \mathrm{O}$ de l'échantillon de fruit d'olivier analysé

\begin{tabular}{ccc}
\hline Élément & $\begin{array}{c}\text { Teneur en \% de matière } \\
\text { sèche }\end{array}$ & $\begin{array}{c}\text { Teneur en g par kg } \\
\text { d'olive* }\end{array}$ \\
\hline $\mathrm{N}$ & 0,96 & 4,272 \\
$\mathrm{P}_{2} \mathrm{O}_{5}$ & 0,224 & 0,996 \\
$\mathrm{~K}_{2} \mathrm{O}$ & 1,368 & 6,087 \\
\hline
\end{tabular}

* : le taux de matière sèche représente $44,5 \%$ du poids frais

Le tableau 3 regroupe les résultats des analyses du compost effectuées pour la détermination des teneurs de ce fertilisant organique en $\mathrm{N}, \mathrm{P}_{2} \mathrm{O}_{5}$ et $\mathrm{K}_{2} \mathrm{O}$.

Tableau 3. Teneurs en $\mathrm{N}, \mathrm{P}_{2} \mathrm{O}_{5}$ et $\mathrm{K}_{2} \mathrm{O}$ du compost

\begin{tabular}{ccc}
\hline Élément & $\begin{array}{c}\text { Teneur en \% de matière } \\
\text { sèche }\end{array}$ & $\begin{array}{c}\text { Teneur en Kg par tonne } \\
\text { de compost frais* }\end{array}$ \\
\hline $\mathrm{N}$ & 1,4 & 10 \\
$\mathrm{P}_{2} \mathrm{O}_{5}$ & 0,79 & 5,5 \\
$\mathrm{~K}_{2} \mathrm{O}$ & 2,64 & 18,5 \\
\hline
\end{tabular}

* : le taux de matière sèche représente $70 \%$ du poids frais

Le tableau 4 présente les résultats des analyses du compost fractionné. On note la différence entre les rapports $\mathrm{C} / \mathrm{N}$ des différentes fractions de compost. Ce qui montre qu'il faut se méfier de la valeur moyenne. Ce rapport est élevé dans le cas de la coque des noyaux. Cette fraction est de nature à diminuer la vitesse de la minéralisation du compost à cause de sa richesse en cellulose et lignine qui résistent à ce processus même après plusieurs années. 
Tableau 4. Teneurs en $\mathrm{N}$ et $\mathrm{C}$ du compost et de ses différentes fractions

\begin{tabular}{cccc}
\hline Échantillon & \% C & \% N & $\mathbf{C} / \mathbf{N}$ \\
\hline $\mathrm{CP}^{*}$ & 31 & 1,40 & 22,14 \\
$\mathrm{CC}^{*}$ & 41 & 0,49 & 83,67 \\
$\mathrm{CF}^{*}$ & 28 & 1,53 & 18,30 \\
\hline
\end{tabular}

*CP : compost complet, CC : fraction constituée par les coques des noyaux des olives et $\mathrm{CF}$ : fraction constituée par le fumier bovin, les fientes de volaille et la pulpe du grignon d'olive

\subsection{Méthodologie de fertilisation biologique}

Par cette fertilisation, on a visé la réalisation des objectifs suivants : (i) maintenir un taux d'humus stable dans le sol, (ii) satisfaire les besoins de l'olivier, (iii) apporter l'engrais biologique à des périodes de forte demande par l'olivier.

Le rendement escompté de l'oliveraie lorsqu'elle atteindra 10 ans d'âge est de $100 \mathrm{~kg}$ d'olives par arbre, c'est-à-dire 20 tonne par hectare et les fertilisants à utiliser sont des fertilisants organiques dans le cadre d'un système de production biologique : arboriculture - élevage.

\subsubsection{Maintien du taux d'humus dans le sol}

Le stock d'humus dans le sol est fluctuant. Pour qu'il reste stable, il faut que les entrées compensent les sorties. Il est donc intéressant de savoir, pour un sol donné : (i) le pourcentage du tonnage de matière sèche d'engrais organique apporté chaque année au sol qui est transformé en humus. Ce pourcentage est le coefficient isohumique $\mathrm{K}_{1}$ qui dépend du type de sol et de la nature de la matière sèche enfouie [17]. Pour le compost utilisé dans cette étude on admet un coefficient isohumique de $25 \%$, à cause de l'existence des particules de coque. (ii) le pourcentage du stock d'humus stable minéralisé par an. Ce pourcentage est le coefficient de minéralisation $\mathrm{K}_{2}$. Pour cette étude, il s'agit d'une oliveraie conduite en intensif avec une irrigation abondante assurant un taux d'humidité suffisant dans le sol associé à une température optimale surtout pendant la saison estivale. La minéralisation est alors à son optimum, on admet un taux de minéralisation $\mathrm{K}_{2}$ de $5 \%$ [22].

Le renouvellement de l'humus détruit est nécessaire pour maintenir un taux acceptable de 
matière organique dans le sol. Ainsi, dans les conditions actuelles de la parcelle d'étude, il y a $1,49 \%$ d'humus stable par hectare de terre, dans la couche 0-30 cm, de masse 3300 tonnes, soit $3300 * 1,49 / 100=49$ tonnes d'humus. Chaque année $5 \%$ de l'humus stable est minéralisé, soit $49 * 5 / 100=2,45$ tonnes d'humus détruits par hectare. Pour renouveler l'humus détruit on dispose de l'amendement humifère (le compost dans notre cas) dont la MS donne $25 \%$ d'humus (coefficient isohumique $\mathrm{k}_{1}$ ). Ainsi, la quantité d'humus nécessaire pour compenser les pertes est de $2,45 * 100 / 25=9,8$ tonnes de MS soit 9,8 $* 100 / 70=14$ tonnes/ha/an de compost frais à taux d'humidité de $30 \%$.

\subsection{2. Évaluation des prélèvements par la récolte et la taille}

En se basant sur les résultats d'analyse de l'échantillon de fruit d'olivier et pour un rendement escompté de $100 \mathrm{~kg}$ par arbre, les exportations des fruits ont été évaluées à 4,270 kg N, $0,996 \mathrm{~kg} \mathrm{P}_{2} \mathrm{O}_{5}$ et $6,087 \mathrm{~kg} \mathrm{~K} \mathrm{~K}_{2} \mathrm{O}$ par tonne d'olive. Comparées aux données de la littérature, ces exportations par les fruits de la variété Picholine se rapprochent de celles enregistrées pour d'autres variétés cultivées dans des conditions pédoclimatiques différentes. Prenons le cas de la zone Kerma où [23] a déterminé les exportations par les fruits d'olivier cultivé en irrigué et donnant un rendement de $45 \mathrm{~kg}$ par arbre. Il a noté les exportations suivantes par tonne d'olives : $\mathrm{N}(5 \mathrm{~kg}) ; \mathrm{P}_{2} \mathrm{O}_{5}(1 \mathrm{~kg}) ; \mathrm{K}_{2} \mathrm{O}(8 \mathrm{~kg})$.

L'olivier subit chaque année une taille de régénération. Les produits (feuilles, brindilles, bois) issus de cette opération exportent des quantités importantes d'éléments nutritifs [23] dont il faut tenir compte lors de l'élaboration du bilan de fumure. Les résultats de Louvrier cité par [23] montrent que la taille peut correspondre à un poids de matière végétale équivalent à celui des fruits, exportant environ la moitié de l'azote et de l'acide phosphorique et le tiers de la potasse. Dans notre cas, l'évaluation des prélèvements par la récolte concerne une oliveraie conduite en irrigué et pour un rendement de $100 \mathrm{~kg}$ par arbre. A terme, et sur la base des observations précédentes, on estime que la taille prélèvera un taux de $50 \%$ en azote et en $\mathrm{P}_{2} \mathrm{O}_{5}$ et de $20 \%$ en $\mathrm{K}_{2} \mathrm{O}$ par rapport au prélèvement total par la récolte. Ceci tout en adoptant l'hypothèse que les prélèvements par les feuilles qui tombent tout le long du cycle végétatif sont négligeables. Ainsi, le prélèvement total par récolte (fruits + taille) et par hectare sera, 
pour un rendement de 20 tonnes/ha : $171 \mathrm{~kg} \mathrm{~N}, 40 \mathrm{~kg} \mathrm{P}_{2} \mathrm{O}_{5}$ et $170 \mathrm{~kg} \mathrm{~K}{ }_{2} \mathrm{O}$.

\subsection{3. Évaluation des principaux apports}

Les fractions assimilables de $\mathrm{N}$, P et $\mathrm{K}$ se trouvent dans le sol sous diverses formes chimiques qui peuvent varier dans le temps. Plusieurs mécanismes influencent la dynamique de ces éléments dans le sol. Il est important de connaître toutes les sources de gain et de pertes (définitive ou temporaire) afin de mieux évaluer les apports nécessaires. Dans cette démarche on a tenu compte de l'évolution de la surface exploitée par les racines de l'olivier. En effet, vers l'âge de 3/6 ans les racines de l'olivier exploitent une surface de $10 \mathrm{~m}^{2}$ soit, pour une densité de plantation de 200 arbres par ha, $20 \%$ de la surface totale (1 ha). Cette surface exploitée évolue vers un pourcentage de 50 \% vers l'âge de 6/10 ans. Au delà de 10 ans, on admet que la surface totale est exploitée (100\%).

\section{Apport d'azote}

On a adopté la méthode du bilan pour évaluer les doses appropriées tenant compte des conditions pédoclimatiques de la zone d'étude et de l'irrigation. Les termes de ce bilan sont: $\mathrm{S}+\mathrm{x}+\mathrm{M}=\mathrm{E}+\mathrm{P}$ ou S représente la quantité d'azote minéral $\left(\mathrm{NH}_{4}{ }^{+}+\mathrm{NO}_{3}{ }^{-}\right)$trouvée dans le sol à la fin du printemps, M est l'azote minéral largué durant la culture par minéralisation de l'azote organique contenu dans l'humus du sol, E est la quantité d'azote exportable par la récolte estimée, $\mathrm{P}$ représente les pertes de $\mathrm{N}$ par volatilisation, lixiviation, blocage, immobilisation biologique, ... et x la quantité d'azote à apporter par le fertilisant pour un rendement de $20 \mathrm{t} / \mathrm{ha}$.

Le début du cycle végétatif a été pris au redémarrage de la végétation soit avant la floraison qui se produit en général vers le mois d'avril. Cette période correspond d'ailleurs à la fin de la saison des pluies, c'est à dire la fin de la période de lixiviation naturelle et le démarrage progressif des irrigations. Les pertes de $\mathrm{N}$ par lixiviation sont donc importantes ce qui engendre un stock faible dans le sol. Sur la base de cette hypothèse et selon l'évolution de la surface exploitée par les racines de l'olivier on a considéré vers l'âge de 3/6 ans un stock initial d'azote (S) dans le sol de $4 \mathrm{~kg}$ dans la couche 0-30 cm, vers l'âge de 6/10 ans un stock 
de $10 \mathrm{~kg}$ et pour un âge supérieur à 10 ans on a considéré un stock initial d'azote de $20 \mathrm{~kg} / \mathrm{ha}$. D'après [24], l'azote est stocké dans le sol sous forme d'humus stable qui dose environ $5 \%$ d'azote organique et corps microbiens. L'azote se minéralise progressivement sous l'action de la flore microbienne, et, au stade ultime de cette évolution une partie de l'azote organique devient sous la forme nitrique, disponible pour la plante. Ainsi en considérant un taux de minéralisation de $5 \%$, la quantité d'azote minéral $\mathrm{M}$ a été estimée à $25 \mathrm{~kg}$ vers l'âge de 3/6 ans, $60 \mathrm{~kg}$ vers $6 / 10$ ans et de $123 \mathrm{~kg}$ pour un âge supérieur à 10 ans.

Les prélèvements E par récolte et par hectare étant de $171 \mathrm{~kg}$ N. Pour des rendements moyens de $10 \mathrm{~kg} /$ arbre vers l'âge de 3/6 ans, de $40 \mathrm{~kg}$ /arbre vers l'âge de 6/10 ans et de $100 \mathrm{~kg} /$ arbre pour un âge supérieur à 10 ans, ces prélèvements seraient respectivement de 35, 90 et $123 \mathrm{~kg}$ d'azote.

L'azote est connu par sa grande mobilité dans le sol [17]. La lixiviation est le principal facteur qui engendre les pertes des nitrates. L'intensité de ce mécanisme dépend du régime hydrique du sol et donc de sa texture et de l'irrigation. La forme nitrique de l'azote n'est pas retenue par le sol, elle se perd par lixiviation. En cas d'apport d'eau au sol, cette fraction suit le front d'humectation. Ainsi, l'application d'une dose de lessivage engendre la perte d'azote par lixiviation. En considérant dans notre cas une fraction de lessivage de 20 \% (l'eau d'irrigation titre de 1 à $2 \mathrm{~g} / \mathrm{l})$ les pertes $(\mathrm{P})$ de $\mathrm{N}$ par ce mécanisme ont été estimées sur cette base à $20 \%$. [25] a signalé que les pertes d'azote sont engendrées, outre par la lixiviation, par la volatilisation, l'immobilisation ou la réorganisation biologique ainsi que le piégeage de la forme $\mathrm{NH}_{4}{ }^{+}$dans les espaces interfoliaires. Pour l'ensemble de ces pertes et pour d'autres formes qui peuvent éventuellement exister, on a adopté un taux de $20 \%$.

Ainsi, le total des pertes de $\mathrm{N}$ a été estimé à $\alpha=40 \%$ par rapport à l'ensemble des sources d'approvisionnement du sol en azote minéral (stock + minéralisation + fertilisant). D'où une grande efficience de l'azote $(60 \%)$ vue qu'on est en présence d'une irrigation localisée (goutte à goutte) bien maîtrisée permettant de minimiser les pertes. Ainsi, $\mathrm{P}=\alpha(\mathrm{S}+\mathrm{x}+\mathrm{M})$ avec $\alpha=40 \%$. La quantité x d'azote à apporter par le fertilisant pour un rendement de 20 t/ha est donnée par : $\mathrm{x}=[\mathrm{E} /(1-\alpha)]-(\mathrm{M}+\mathrm{S})=(171 / 0,6)-(73,755+30)=133 \mathrm{~kg} \mathrm{~N} / \mathrm{ha}$. En considérant l'évolution de la surface exploitée l'apport d'azote serait de $27 \mathrm{~kg}$ vers l'âge de 
3/6 ans, de $66 \mathrm{~kg}$ vers l'âge de 6/10 ans et de $133 \mathrm{~kg}$ vers un âge supérieur à 10 ans.

\section{Apport phospho-potassique}

Le sol étudié étant bien pourvu en $\mathrm{P}_{2} \mathrm{O}_{5}$ et $\mathrm{K}_{2} \mathrm{O}$ (respectivement 22 ppm et 288 ppm). Il suffira alors d'entretenir la richesse du sol par des apports de fertilisants majorés par des facteurs de correction par rapport aux prélèvements du fait que ces éléments sont sujets à différentes formes de pertes.

Élément très peu mobile, les pertes du phosphore par lixiviation sont très faibles si non négligeables [17]. La disponibilité de cet élément dépend du taux de calcaire actif et du taux d'argile présents dans le sol. Plus ces taux sont élevés plus la disponibilité de P pour la plante est faible. Dans notre cas le sol est riche en ces deux éléments $\left(\mathrm{CaCO}_{3}=26 \%\right.$ et $\left.\mathrm{A}=40 \%\right)$ ce qui rend l'efficience de $\mathrm{P}$ très faible. On admet en régime irrigué que l'efficience du phosphore du sol serait meilleure qu'en régime pluvial [26]. Ainsi, on a admis que $10 \% \mathrm{du}$ $\mathrm{P}_{2} \mathrm{O}_{5}$ est disponible pour l'arbre. Les prélèvements ont été majorés par un facteur de correction de $1+(100-10) / 100=1,9$. Ainsi, l'apport de $\mathrm{P}_{2} \mathrm{O}_{5}$ a été estimé à $40 * 1,9=76 \mathrm{~kg} / \mathrm{ha}$. En considérant l'évolution de la surface exploitée cet apport a été estimé à $15 \mathrm{~kg}$ vers l'âge de 3/6 ans, de $40 \mathrm{~kg}$ vers l'âge de 6/10 ans et de $76 \mathrm{~kg}$ vers un âge supérieur à 10 ans.

Concernant le potassium, le principal processus causant la perte de cet élément est son blocage entre les feuillets d'argile ([17]). Cette perte dépend de la teneur du sol en argile et du type d'argile présent ; elle est désignée par la capacité de fixation de K dans le sol. Plusieurs formules sont utilisées pour estimer le taux de potassium perdu dans le sol telle que celle de Quenemer : $\mathrm{CF}=\% \mathrm{~A}+15$, où $\mathrm{CF}$ représente la capacité de fixation en $\%$ d'argile dans le sol. Dans notre cas, avec un taux d'argile $\mathrm{A}=40 \%$ donc $\mathrm{CF}=55 \%$ de $\mathrm{K}$ sera fixé. Les prélèvements en $\mathrm{K}_{2} \mathrm{O}$ ont été donc corrigés par un facteur de $1+55 / 100=1,55$. D'ou l'apport nécessaire en $\mathrm{K}_{2} \mathrm{O}$ a été estimé à $170 * 1,55=264 \mathrm{~kg} /$ ha. En considérant l'évolution de la surface exploitée l'apport de potassium a été estimé à $53 \mathrm{~kg}$ vers l'âge de $3 / 6$ ans, de $132 \mathrm{~kg}$ vers l'âge de 6/10 ans et de $264 \mathrm{~kg}$ vers un âge supérieur à 10 ans.

Les apports nécessaires en $\mathrm{N}, \mathrm{P}_{2} \mathrm{O}_{5}$ et $\mathrm{K}_{2} \mathrm{O}$ sont regroupés dans le tableau 5. 
Tableau 5. Apports nécessaires en $\mathrm{N}, \mathrm{P}_{2} \mathrm{O}_{5}$ et $\mathrm{K}_{2} \mathrm{O}$ selon l'âge de l'oliveraie

\begin{tabular}{ccccccc}
\hline \multirow{2}{*}{ Age } & Rendement & Surface & \multicolumn{3}{c}{ Apports (kg) } \\
\cline { 4 - 6 } & $(\mathbf{k g} /$ arbre) & exploitée (\%) & $\mathbf{N}$ & $\mathbf{P}_{\mathbf{2}} \mathbf{O}_{\mathbf{5}}$ & $\mathbf{K}_{\mathbf{2}} \mathbf{O}$ \\
\hline $\mathbf{3 / 6}$ ans & 10 & 20 & 27 & 15 & 53 \\
$\mathbf{6 / 1 0}$ ans & 40 & 50 & 66 & 40 & 132 \\
$>\mathbf{1 0}$ ans & 100 & 100 & 133 & 76 & 264 \\
\hline
\end{tabular}

\subsubsection{Doses de fertilisants à apporter}

Pour apporter les quantités nécessaires de $\mathrm{N}, \mathrm{P}_{2} \mathrm{O}_{5}$ et $\mathrm{K}_{2} \mathrm{O}$, on dispose du compost biologique localement préparé tout en tenant compte de l'apport des éléments nutritifs par minéralisation de l'humus du sol qui contient des éléments nutritifs sous forme organiques libérés progressivement par minéralisation. La teneur de l'azote sous forme organique dans l'humus a été estimée, comme mentionné précédemment à $5 \%$. La participation de l'humus du sol dans l'apport de cet élément a été prise en compte lors de l'élaboration de la méthode du bilan. Pour le phosphore, une partie des besoins de l'arbre en cet élément est satisfaite par l'humus du sol contenant une teneur de $1 \%$ de $\mathrm{P}$ organique soit $500 \mathrm{~kg}$ par hectare. Cette quantité sera minéralisée pour donner $15 \mathrm{~kg}$ de $\mathrm{P}$ minéral disponible à la plante soit une quantité de $34 \mathrm{~kg}$ de $\mathrm{P}_{2} \mathrm{O}_{5}$. Les teneurs du potassium dans l'humus du sol sont négligeables.

Concernant le compost, on a supposé que la totalité des éléments N, P et K s’y trouve sous la forme organique. Enfoui dans le sol, ce fertilisant subit une minéralisation primaire à raison de $75 \%$ au cours de la première année, le reste est supposé donner de l'humus qui se minéralise à raison de $5 \%$ par an sous régime irrigué, en supposant que ce compost est répandu sous les arbres et localisé dans la zone des goutteurs. En outre, ce raisonnement suppose que la dynamique de $\mathrm{P}$ et de $\mathrm{K}$ apportés sous forme organique et libérés par minéralisation soit de même type que celle qui caractérise $\mathrm{P}$ et $\mathrm{K}$ des engrais chimiques. Il est probable que cette hypothèse ne soit pas vérifiée car la minéralisation se produit progressivement toute l'année, et de ce fait les pertes sont plus faibles.

Pour un rendement escompté de $100 \mathrm{~kg}$ par arbre on doit apporter une quantité de compost qui satisfait les besoins de l'arbre évalués précédemment par le biais des prélèvements. Ainsi, 
le besoin en azote de $133 \mathrm{~kg} /$ ha est apporté par une quantité de compost frais de (133/10) * $100 / 75=18$ tonnes qui, par minéralisation, libère la quantité nécessaire d'azote minéral disponible pour la plante. Cette quantité apporte aussi $93 \mathrm{~kg}$ de $\mathrm{P}_{2} \mathrm{O}_{5}$ organique et $314 \mathrm{~kg}$ de $\mathrm{K}_{2} \mathrm{O}$ organique soit respectivement $75 \mathrm{~kg}$ et $251 \mathrm{~kg}$ sous forme minérale. Ces teneurs permettent de satisfaire les besoins de l'olivier en ces deux éléments qui sont respectivement de 76 et de $264 \mathrm{~kg}$ par hectare à l'horizon de la $10^{\text {ème }}$ année pour une production de 20 tonnes à l'hectare.

Cette quantité de compost nécessaire pour satisfaire les besoins nutritifs de l'olivier va aussi permettre de renouveler les pertes d'humus du sol par minéralisation en laissant une quantité d'humus de $18 * 25 / 100=4,5$ tonnes (25 étant le coefficient isohumique $\mathrm{k}_{1}$ ). En totalité et tenant compte des pertes possibles, l'apport nécessaire de compost a été estimé à 20 tonnes/ha/an. Selon l'évolution de la surface exploitée par l'olivier les apports nécessaires de compost ont été estimés à $(27 / 10)^{*} 100 / 75=4$ tonnes vers l'âge de $3 / 6$ ans, de $(66 / 10) * 100 / 75=10$ tonnes vers l'âge de $6 / 10$ ans et de $(133 / 10) * 100 / 75=20$ tonnes pour un âge supérieur à 10 ans.

Ainsi les quantités de fertilisants à apporter étant déterminées, il faut donc bien choisir le lieu et la date d'apport pour augmenter l'efficience de ces fertilisants et limiter au maximum leur gaspillage.

\subsubsection{Périodes d'apport}

Il est possible d'administrer le fertilisant organique en une seule fois pendant le cycle végétatif de l'arbre et ça sera au début de son cycle (janvier-février). Cette modalité présente l'inconvénient qu'il y a un risque de pertes des éléments nutritifs par lixiviation sous l'effet de la pluie (surtout pour l'azote). Il sera donc plus utile d'apporter le compost en deux fois pendant le cycle végétatif. Les courbes du diagnostic foliaire montrent l'existence d'un point minimum vers les mois de juillet-août. La fumure organique devra tendre à élever cette teneur à une valeur maximum. Après cette chute la courbe augmente, l'olivier entre dans la phase de fructification qui demande la disponibilité des éléments nutritifs pour assurer une bonne production. 
On préconise la répartition de la fumure organique en deux fractions :

- La moitié de la quantité de compost sera donnée pour satisfaire les besoins de l'olivier pendant la période du réveil printanier (février-mars) qui se manifeste par l'apparition de nouvelles pousses terminales et l'éclosion des bourgeons axillaires et aussi pendant la phase de la floraison. Une bonne alimentation de l'arbre pendant cette période est nécessaire pour assurer un bon rendement puisque de la formation des fleurs va dépendre la future récolte.

- La deuxième moitié pendant la phase qui succède la chute estivale c'est à dire juste après la nouaison (juin). L'olivier a besoin des éléments nutritifs pendant cette période caractérisée par le développement et le grossissement des fruits. Durant cette phase de grossissement du fruit se produit une chute des olives due essentiellement à des insuffisances alimentaires. L'apport de fertilisant pendant cette période pourra donc limiter cette chute et assurer de ce fait un bon rendement.

On recommande les doses de compost regroupées dans le tableau 6 des apports nécessaires suivant l'évolution de la surface exploitée par l'olivier et selon les deux modalités d'apport :

Tableau 6. Doses de compost selon l'évolution de la surface exploitée par l'olivier et selon deux modalités d'apport.

\begin{tabular}{|c|c|c|c|c|}
\hline \multirow{2}{*}{ Age } & \multirow{2}{*}{$\begin{array}{c}\text { Surface exploitée } \\
(\%)\end{array}$} & \multicolumn{3}{|c|}{ Apport Compost (t / S exp.) } \\
\cline { 3 - 5 } & & Modalité 1 & \multicolumn{2}{|c|}{ Modalité 2 } \\
\cline { 3 - 5 } & 20 & 4 & 2 & 2 \\
\hline $3 / 6$ ans & 50 & 10 & 5 & 5 \\
\hline $6 / 10$ ans & 100 & 20 & 10 & 10 \\
\hline$>10$ ans & & & & juin-fév \\
\hline
\end{tabular}

\section{CONCLUSION}

L'élaboration d'une méthodologie de fertilisation dans le cadre d'un système d'oléiculture biologique s'avère une opération complexe car elle doit intégrer un ensemble de données relatives à la fertilité du sol, aux exigences nutritives de l'olivier, au rendement escompté ainsi 
qu'à la qualité et à la quantité de fertilisants biologiques disponibles. En prenant en considération tous ces facteurs, un program de fertilisation est élaboré lors de la présente étude qui pourra guider les oléiculteurs tunisiens vers l'établissement d'une fumure biologique adaptée aux conditions de leurs exploitations. Enfin, bien que ce travail ne s'intéresse qu'à l'aspect fumure, il est utile de rappeler qu'on ne pourra pas dissocier cet aspect de toutes les autres pratiques agronomiques en matière d'oléiculture. La production étant la résultante d'une combinaison coordonnée et harmonieuse de ces différents facteurs.

\section{REMERCIEMENTS}

Les auteurs tiennent à remercier l'agriculteur M. Turki d'avoir accepter la réalisation de cette étude dans son olivereaie ainsi que le personnel du laboratoire de Sciences du Sol et Environnement de l'Institut National Agronomique de Tunisie.

\section{REFERENCES}

[1] Ifoam, Fibl, Growing organic agriculture sector explores its future, BioFach, 2014, Istanbul, pp. 8.

[2] Patil S., Reidsma P., Shah P., Purushothaman S., Wolf J, Comparing conventional and organic agriculture in Karnataka, India: Where and when can organic farming be sustainable? Land Use Policy 37, 2014, pp. 40-51.

[3] Bjørkhaug H., Blekesaune A, Development of organic farming in Norway: A statistical analysis of neighbourhood effects. Geoforum 45, 2013, pp. 201-210.

[4] Gopal B.T. and Kanokporn R, Adoption and extent of organic vegetable farming in Mahasarakham province, Applied Geography, Thailand, 31, 2011, pp. 201-209.

[5] DG/ DPIA. Direction Générale de Développement et de Planification des Investissements Agricoles, Tunisie. S/D Statistiques agricoles, 2014. Résultats de l'enquête sur le suivi de la campagne agricole.

[6] Fernández-Escobar R., Parra M.A., Navarro C and Arquero O, Foliar diagnosis as a guide to olive fertilization. Spanish Journal of Agricultural Research, 2009, 7(1), pp. 212-223.

[7] Fernandez-Hernandez A., Beltran G., Fernandez-Escobar R, Floral analysis cannot be 
considered as an alternative to the foliar diagnosis in the olive. Scientia Horticulturae, 112, 2007, pp. 23-26.

[8] Ibrahimi K, Fertilité du sol et fertilisation de l'olivier: Elaboration d'un conseil de fumure biologique pour une oliveraie au Nord-ouest de la Tunisie, Éditions Universitaires Européenes, 2013, pp. 104.

[9] Loussert R., and Brousse G, Techniques agricoles et productions méditerranéennes, L'olivier, Paris, Maisonneuve et Larose, 1978, pp. 465.

[10] Bonneau M and Souchier B, Constituants et propriétés du sol. Paris, Masson, 1979, pp. 459.

[11] Baize D, Guide des analyses courantes en pédologie : choix expression présentation interprétation, Paris, INRA, 1988, pp. 172.

[12] Musy A., and Soutter M, Physique du sol. Première édition. Presses polytechniques et universitaires normandes, 1991, pp. 335.

[13] Pauwels J.M., Van Ranst E., Verloo M., Mvondoze, Manuel de laboratoire de pédologie, Bruxelles, Publications agricoles, 1992, pp. 265.

[14] Duchaufour P, Pédologie : Sol, végétation, environnement. 3 ème édition, Paris, Masson, 1991, pp. 289.

[15] CPCS, Classification des sols. Commission de Pédologie et de la Cartographie des Sols, Grignon, INRA, 1967, pp 87.

[16] Wandruszka R, Phosphorus retention in calcareous soils and the effect of organic matter on its mobility. Geochem Trans., 2006, 7, 6.

[17] Soltner D, Les bases de la production végétale: le sol, $18^{\text {ème }}$ édition. Sainte-Gemmes-Sur-Loire, Angers, Collection sciences et techniques agricoles, 1990, pp. 467. [18] Zaier M, Appréciation de la fertilité phospho-potassique du sol : problèmes et normes d'interprétation, Tunis, Direction des sols, 1988, pp. 86.

[19] Recalde L, Fertilisation. Deuxième Séminaire Oléicole International. Cordoue, Espagne, 1975, pp. 43-64.

[20] Gargouri K., Mhiri A, Relationship between soil fertility and phosphorus and potassium olive plant nutrition. Série A. Séminaires Méditerranéens; 50, 2002, pp. 199-204. 
[21] Llamas J.F, Basis of fertilization in olive cultivation and the olive tree's vegetative cycle and nutritional needs, Int. Course on the fertilization and intensive cultivation of the olive, 1983, Cordoba, Spain, 1984, pp. 1-25.

[22] Mhiri A, Notes sur la fertilisation des céréales en Afrique du Nord, Stage Med-Céréales, Tunisie, 1995, pp. 1-9.

[23] Hutter W, Fertilisation de l'olivier, état de recherches. Séminaire oléicole nationale. Sfax, Tunisie, 1970, 1-13.

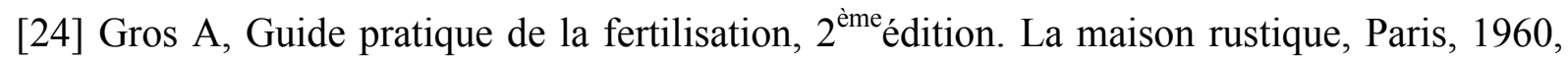
pp. 406.

[25] Carrante V, Théorie et pratique de la fumure de l'olivier. Inf. Olei. Inter. Revue officielle de la F. I. O, 1969, pp. 24.

[26] Ben Zina N, Fertigation à l'acide phosphorique en sols calcaires. Thèse de doctorat en sciences biologiques appliquées. Faculteit Landbonwkundige en Toegepaste Biologische wetenschappen, Belgique, 1999.

\section{How to cite this article:}

Ibrahimi K and Gaddas F. Organic fertilization of olive tree based on soil analysis and foliar diagnosis: a case study of an olive orchard in the northwest of Tunisia. J. Fundam. Appl. Sci., 2015, 7(3), 322-339. 